\title{
Coherent anti-Stokes Raman Scattering Microscopy
}

\author{
Michiel Müller ${ }^{[b]}$ and Andreas Zumbusch*[a]
}

\begin{abstract}
Coherent anti-Stokes Raman scattering (CARS) microscopy is presented as a new nonlinear optical technique. The combination of vibrational spectroscopy and microscopy allows highly sensitive investigations of unlabelled samples. CARS is an ideal tool for studying a broad variety of samples. The main drawback of the technique is its non-zero-background nature, which implies that the signal has to be detected against a nonresonant background. The need to solve this problem is reflected in the rapid technolog-
\end{abstract}

ical developments that have been observed during the last decade. Recent results show that CARS microscopy has the potential to become an important complementary technique that can be used with other well-established microscopic methods. Although it has some limitations, it offers unique access to many problems that cannot be tackled with conventional techniques. For this reason, it can be expected that the impressive growth of the field will continue.

\section{Introduction}

Optical microscopy has long been a key technology for materials science and biological research. Many problems could only be solved after the introduction of suitable microscopic techniques. This fact is most obvious in biology. The discovery of biological cells and the emergence of cell biology as a scientific discipline in the 17th and 18th centuries is intimately intertwined with the invention and development of optical microscopy. By constructing the first optical microscopes and using them for the observation of biological samples, researchers such as Hooke and van Leeuwenhoek laid the foundations for both fields. This close interdisciplinary exchange continued in the 19th century when the focus of development shifted to the introduction of histological staining methods, using the newly invented artificial dyes. Gram's staining of bacteria is perhaps the most prominent among these methods today. Staining allows the specific visualization of microscopic structures based on their molecular composition and the resulting ability to bind dyes. The technique yields a strong image contrast and, since most untreated samples absorb light only weakly, it is tremendously successful. Unfortunately, however, staining sacrifices non-invasiveness which is one of the main advantages of optical microscopy. Non-invasiveness is of pivotal importance in studies of live samples, and also for samples which cannot be stained, as is the case for most material scientific specimens. This problem initiated a quest for optical microscopy techniques which provide contrast without the necessity to stain the sample with a chromophore. Such an advantage is exactly provided by phase contrast microscopy and differential interference contrast microscopy, which were invented by Zernike and Nomarski, respectively, in the first half of the 20th century. Both methods rely on small differences in the refractive index of the sample. The phase shifts, which are observed after the excitation light has transversed the sample, are used to generate the image contrast. From this mechanism it is clear that neither of the two techniques is chemically selective.

Today, the techniques described above are used as complementing methods in the laboratory: label-free investigations are performed using phase contrast and differential interference contrast microscopy or other less-widespread methods such as, for example, polarization microscopy. Staining, on the other hand, allows experiments in which specific structures can be visualized with high sensitivity. Although these more traditional microscopy methods are important tools, modern technological developments have brought a broad variety of new microscopy technologies during the last two decades. The main driving forces behind this wave of innovation have been the commercial availability of continuous-wave and pulsed lasers as excitation sources, the advent of new labeling techniques (especially using autofluorescent proteins, AFP), and fast data-processing equipment. All three points have contributed to the final breakthrough of confocal laser scanning fluorescence microscopy, with which distributions of fluorophores can be visualized in three dimensions. ${ }^{[1]}$ The huge impact of this technique on cell biology is mainly owed to the introduction of green fluorescent protein variants as genetically encoded fluorophores. ${ }^{[2]}$ By expressing a fusion protein composed of an AFP with a target protein in living cells and organisms, ultimate specificity can be achieved: proteins can now be tracked by monitoring the fluorescence of the AFP. However,

\section{[a] Prof. A. Zumbusch}

Department Chemie and Center for Applied Photonics

Universität Konstanz, Fach M722, 78457 Konstanz (Germany)

Fax: $(+49) 7531883870$

E-mail:andreas.zumbusch@uni-konstanz.de

[b] Dr. M. Müller

Swammerdam Institute for Life Sciences

University of Amsterdam, P.O. Box 94062

1090 GB Amsterdam (The Netherlands) 
one has to keep in mind that the fusion with an AFP may lead to major changes in the physiological properties of a target protein, which is often smaller than-or of the same size asthe label. In addition to its three-dimensional imaging capability and high specificity, laser scanning fluorescence microscopy offers the advantage that-neglecting other sources of background signal, such as Raman scattering-the spectral shift of the fluorescence signal makes it a zero-background detection scheme. Fluorescence microscopy has indeed become the method of choice for the optical detection of single molecules. ${ }^{[3-5]}$ Apart from the need to stain the sample, the main problem encountered in fluorescence microscopy is the low average survival time of a fluorophore under typical excitation conditions. Electronic excitation in the visible spectral region is always accompanied by photochemical side reactions which modify the fluorophore in such a way that it does not absorb or fluoresce any more. This photobleaching phenomenon severely limits the overall observation time.

\section{Dr. Michiel Müller received his PhD (Thesis: Time-resolved Raman micro- scopy in simple liquids) in physical chemistry from the University of Am- sterdam (The Netherlands) in 1990. In 1994 he joined the Confocal Microsco- py Group led by Prof. Dr. G. J. Brake- nhoff at the University of Amsterdam. In 1997 he received the SEN award- an award issued by the Dutch Micro- scopical Society-for "exceptionally in- novative and productive research by a young scientist". Currently, he is an associate professor in biophys- ics at the Swammerdam Institute for Life Sciences (University of Amsterdam). The research in his "Biophysics and Microscopy Group" focuses on the study of structure/function relationships in (bio)membranes and the development of novel microscopic tech- niques including coherent anti-Stokes Raman scattering (CARS) mi- croscopy and third-harmonic-generation (THG) microscopy.}

Prof. Andreas Zumbusch studied chemistry and physics in Munich (Germany) and Bordeaux (France). He received his $\mathrm{PhD}$ in inorganic chemistry from the University of Karlsruhe. Following this, he did postdoctoral research on nonlinear optical microscopy at the Pacific Northwest National Laboratory (Richland, WA, USA). In 1999 he joined the University of Munich where he was working on the development and application of opti-

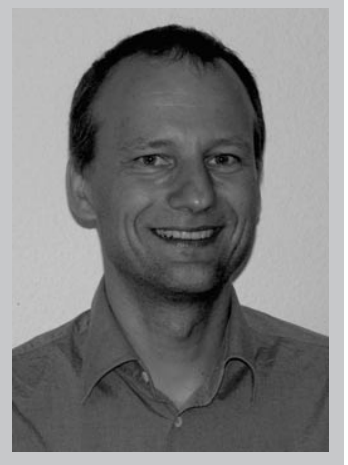
cal single-molecule detection and coherent anti-Stokes Raman scattering (CARS) microscopy. Since 2006 he is a professor for physical chemistry at the University of Konstanz (Germany). His research focuses on the analysis of spectral and spatial dynamics of molecules in (bio)polymers and biological cells and on the development of the relevant optical microscopy techniques.
Despite the overwhelming success of confocal fluorescence microscopy, the problems caused by both photobleaching after electronic excitation and specimen modification by labeling, motivates the search for new optical microscopy techniques that can deliver highly specific signals without prior labeling of the specimen. The relative ease of generating laser pulses with durations of 100 femtoseconds ( $\mathrm{fs}$ ) to a few picoseconds (ps) made it possible to explore nonlinear optical effects as means of contrast generation in label-free microscopy. The most straightforward of these approaches rely on the detection of the second-harmonic $(\mathrm{SHG})^{[6-8]}$ or the third-harmonic generation (THG) of the excitation beam..$^{[9-11]}$ Whereas SHG is surface-specific and requires a local break of the inversion symmetry, THG is sensitive to interfaces in either third-order nonlinear susceptibility and/or refractive index. In both cases, the signal-generation process is commonly nonresonant. Thus, although both techniques work without the use of any external label, they do not provide contrast with chemical selectivity. The latter can be obtained by monitoring the vibrational spectra of the sample molecules. Traditionally, this is achieved by using either direct infrared absorption or Raman scattering microscopy. Both methods, however, have certain limitations which prohibit their use in many applications. IR microscopy suffers from the fact that long wavelengths are used for the excitation. Therefore, the diffraction-limited spot sizes are large and the corresponding optical resolution is low. This problem is further aggravated by the fact that reflective optics with low numerical apertures have to be used. A second problem met with IR microscopy is the broad water absorption which makes investigations in aqueous solutions and in many biological samples difficult or impossible. Spontaneous Raman scattering microscopy avoids these problems because lasers operating in the visible spectral range can be used for the excitation. Raman microscopy has, indeed, been used extensively for material scientific and biological applications. ${ }^{[12]}$ The drawback of this technique is related to the generally low Raman scattering cross-sections. For this reason, high excitation intensities and long integration times have to be used, and this is not tolerated by many samples. In addition, background fluorescence from the sample causes major problems because the Stokesshifted fluorescence is observed in the same spectral range as the (commonly much weaker) Raman signal.

The aim of this Review is to present coherent anti-Stokes Raman scattering (CARS) microscopy as a new tool to generate molecularly specific contrast based on the vibrational spectra of the sample molecules. ${ }^{[13,14]}$ Because of the vibrational contrast generation, no staining of the samples is necessary. In contrast to spontaneous Raman scattering microscopy, CARS microscopy, as a coherent technique, has the advantage that stronger signals are obtained and-most importantly-that the signals are observed at higher energies relative to the excitation wavelengths. For this reason, the fluorescence background from the sample does not interfere with the signal detection. It will be shown that because of these advantages, CARS microscopy has become an attractive technique for microscopic investigations of a broad variety of samples. 


\subsection{Physical Basis of the CARS Process}

CARS is a four-wave mixing process in which a pump, a Stokes, and a probe field with frequencies $\omega_{\mathrm{p},} \omega_{\mathrm{s}}$ and $\omega_{\mathrm{pr}}$ induce a polarization $\mathbf{P}^{(3)}$ in the sample. This oscillating-induced polarization gives rise to an anti-Stokes radiation, with a frequency $\omega_{\mathrm{as}}=\omega_{\mathrm{p}}+\omega_{\mathrm{pr}}-\omega_{\mathrm{s}}$ (Figure 1). Commonly, experiments are per-

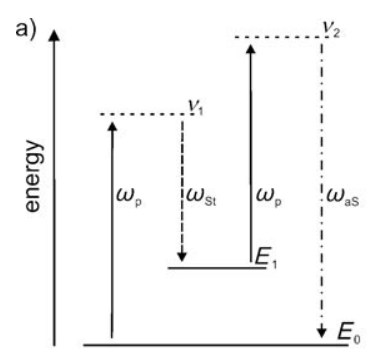

b)

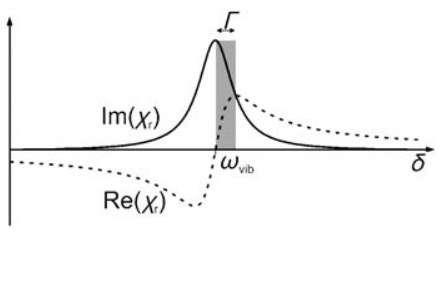

c)
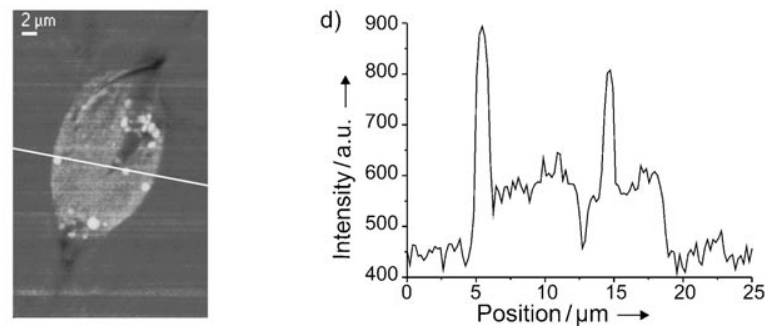

Figure 1. a) CARS level scheme. b) Real (----) and imaginary (-) contributions to the Raman line with a line width of $\Gamma$. c) Image of the lipid distribution inside an HeLa cell as an example for contrast generation with CARS microscopy. The image was recorded at a vibrational resonance of the lipid $\mathrm{CH}_{2}$ stretching vibration at $2845 \mathrm{~cm}^{-1}$. The image size is $26 \times 41.2 \mu \mathrm{m}^{2}$. d) Profile plot along the white line in (c).

formed in a frequency-degenerate manner with the pump and the probe field being obtained from the same laser, so that $\omega_{\text {as }}=2 \omega_{\mathrm{p}}-\omega_{\mathrm{s}}$.

The intensity of the CARS signal is proportional to the square modulus of the induced polarization $I_{\text {CARS }} \propto\left|\mathbf{P}^{(3)}\right|^{2}$. The magnitude of $\mathbf{P}^{(3)}$ itself is determined by the field strength $\mathbf{E}$ of the excitation fields and the nonlinear optical susceptibility of the material $\chi^{(3)}$. It is convenient to decompose $\chi^{(3)}$ into a resonant and a nonresonant part, namely, $\chi_{\mathrm{R}}^{(3)}$ and $\chi_{\mathrm{NR}}^{(3)[15]}$ [Eq. (1)]:

$\mathbf{P}^{(3)}=\left(\chi_{R}^{(3)}+\chi_{N R}^{(3)}\right) \mathbf{E}_{p} \mathbf{E}_{p} \mathbf{E}_{S}^{*}$

In the absence of electronic resonances and for all parallel polarization conditions, both CARS and spontaneous Raman scattering provide, in principle, the same information. ${ }^{[16]}$ Spontaneous Raman scattering is in fact closely related to $\operatorname{Im}\left[\chi^{(3)}\right]$, which describes the CARS vibrational response, although $\chi^{(3)}$ contains additional terms, which do not contribute to the Raman spontaneous emission process. ${ }^{[17]}$ Here, we closely follow the notation of Mukamel and describe spontaneous Raman by an effective third-order susceptibility $\chi_{\text {CARS }}{ }^{[17]}$

The CARS spectral shape, however, differs from the Raman lineshape because of the interference between the contribu- tions from various vibrational modes and the nonresonant background. The value of $\chi_{R}{ }^{(3)}$ is given by Equation (2):

$\chi_{\mathrm{R}}^{(3)}=\sum_{\mathrm{j}} \frac{R_{\mathrm{j}}}{\Omega_{\mathrm{j}}-\left(\omega_{\mathrm{p}}-\omega_{\mathrm{s}}\right)-i \Gamma_{\mathrm{j}}}$

This is a complex sum over all vibrational resonances $j$ involved, with eigenfrequencies $\Omega_{\mathrm{j}}$, oscillator strengths $R_{\mathrm{j}}$, and line widths $\Gamma_{\mathrm{j}}$. Its real part has a dispersive lineshape, while the imaginary part, which mirrors the spontaneous Raman line, has a Lorentzian shape (Figure 1). It follows that [Eq. (3)]:

$I_{\text {CARS }} \propto\left|\chi_{\mathrm{R}}{ }^{(3)}+\chi_{\mathrm{NR}}{ }^{(3)}\right|^{2}=\left|\chi_{\mathrm{R}}{ }^{(3)}\right|^{2}+2 \chi_{\mathrm{NR}}{ }^{(3)} \operatorname{Re}\left\{\chi_{\mathrm{R}}{ }^{(3)}\right\}+\left|\chi_{\mathrm{NR}}{ }^{(3)}\right|^{2}$

As a consequence of the complex nature of $\chi^{(3)}$ and the interference between the resonant and nonresonant parts, CARS typically shows a dispersive lineshape. Compared to the spontaneous Raman spectrum, the vibrational resonances exhibit apparent frequency shifts and differences in the relative peak amplitude.

From this brief description of the basics of the CARS process, the main features of CARS microscopy can be derived. The contrast mechanism in CARS microscopy is provided by the vibrational resonances of $\chi^{(3)}$. If the frequency difference between pump and Stokes, $\omega_{\mathrm{p}}-\omega_{\mathrm{s}}$, coincides with the frequency of a molecular vibration $\Omega_{\mathrm{j}}$ of the sample, then the resonance of $\chi_{R}{ }^{(3)}$ leads to a strong enhancement of the CARS signal. To map the distribution of a molecular species with a vibrational resonance at $\Omega_{\mathrm{j}}$, the frequency difference $\omega_{\mathrm{p}}-\omega_{\mathrm{s}}$ is tuned to $\Omega_{\mathrm{j}}$ and the CARS signal is monitored while scanning the laser beams over the sample (Figure 1). Since the signal generation is a third-order nonlinear optical process, an efficient CARSsignal generation necessitates high excitation intensities, which are only obtained in the focal volume of a tightly focused pulsed laser beam. Therefore, in analogy to two-photon microscopy, ${ }^{[18]}$ CARS microscopy offers a three-dimensional imaging capability.

An important advantage of CARS microscopy over spontaneous Raman scattering microscopy is the fact that background fluorescence from the sample does not interfere with the CARS signal detection. Since $\omega_{\mathrm{p}}>\omega_{s}$, it follows that the frequency $\omega_{\text {as }}$ is higher than all excitation frequencies. This also implies that all background fluorescence and spontaneous Raman signals from the sample will be observed at lower frequencies compared to that of the excitation.

In contrast to fluorescence microscopy, however, the signal in CARS microscopy is not detected against a zero-background. This issue is attributed to the nonresonant part of the susceptibility $\chi_{\mathrm{NR}}{ }^{(3)}$, which has a purely electronic origin. In the absence of electronic resonances, $\chi_{\mathrm{NR}}{ }^{(3)}$ shows almost no frequency dependence. However, the signal is enhanced if an electronic resonance of the pump beam coincides with a two-photon transition in the sample. ${ }^{[19]}$ The existence of the nonresonant background is the main obstacle encountered in practical applications of CARS microscopy since it severely limits the achievable sensitivity. For this reason, most of the efforts during the last 
years have been invested in suppressing the nonresonant background or turning it into an advantage.

\section{Experimental Realizations of CARS Microscopy}

The basis for CARS microscopy is CARS as a spectroscopic technique. CARS spectroscopy, as established by Maker and Terhune in the mid-60s, ${ }^{[20]}$ has found a broad variety of applications. Many of these concern high-resolution spectroscopic studies in the gas and condensed phases. Although the theoretical description of the physical processes in CARS spectroscopy and microscopy remains the same and many of the spectroscopic variants of CARS can be adapted to microscopy, the recent development of CARS microscopy has shown that the experimental requirements for CARS microscopy differ greatly from those for CARS spectroscopy. The main differences concern the excitation geometry and the laser sources as described in the following.

\subsection{Excitation Geometry}

Because of the coherent nature of the CARS process, a strong signal is only obtained if constructive interference is maintained between the beams within the interaction volume. The corresponding phase-matching condition is $|\Delta \mathbf{k}| I \ll \pi$, with the interaction length $l$, the wave vector mismatch $\Delta \mathbf{k}=$ $\mathbf{k}_{\mathrm{as}}-\left(2 \mathbf{k}_{\mathrm{p}}-\mathbf{k}_{\mathrm{s}}\right)$, and the wave vectors $\mathbf{k}_{\mathrm{as}}, \mathbf{k}_{\mathrm{p}}$, and $\mathbf{k}_{\mathrm{s}}$ of the antiStokes, the pump, and the Stokes fields, respectively (Figure 2).

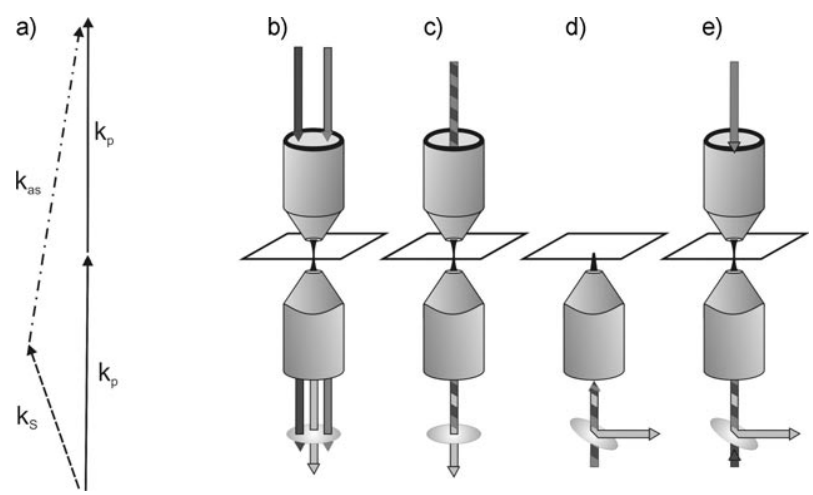

Figure 2. a) Phase-matching scheme commonly employed in CARS spectroscopy. A variety of excitation geometries is used: b) phase-matched excitation c) collinear excitation, d) epi-detection, and e) counterpropagating also used in a wide-field geometry.

In condensed-phase CARS spectroscopy, dispersion leads to a large phase mismatch and, consequently, to low CARS signals if a collinear excitation geometry is employed. This problem is solved in CARS spectroscopy by choosing an appropriate angle between the exciting laser beams. For the first CARS microscopy experiment, this scheme of laterally displacing the two excitation beams before focusing was employed. ${ }^{[21]}$ The CARS signal can then be either spectrally filtered or spatially separated from the excitation light. ${ }^{[22]}$ Although microscopic images can be produced in this fashion, the need to displace the excitation beams relative to each other severely reduces the effective numerical apertures and therefore also the achievable spatial resolution. This obvious problem hampered the further development of CARS microscopy after its first demonstration.

CARS microscopy saw a renaissance when it was realized that the phase-matching conditions were fulfilled in a collinear excitation geometry under tight focusing conditions ${ }^{[23]}$ (Figure 2). Focusing excitation lasers with high-numerical-aperture lenses onto diffraction-limited spot sizes reduces the interaction length to a few microns. This and the large cone of wave vectors present in the tightly focused beams lead to a fulfillment of the phase-matching condition and, consequently, to a strong CARS signal. For structures that are significantly larger than the wavelengths involved, the CARS signal almost exclusively propagates in the forward direction. This means that also the nonresonant CARS signal from the surrounding medium will be collected in this direction, a fact that can put severe limitations on the achievable signal-to-background ratios.

A detailed theoretical treatment of the CARS signal generation and propagation shows that comparatively strong CARS signals can also be obtained in the backward direction ${ }^{[24-26]}$ (Figure 2). This is the case when the contributing structural features are of sizes much smaller than the excitation wavelengths. The phase-matching condition is then fulfilled in all directions and the CARS signal is radiated more or less isotropically. For structures of growing sizes the signal is increasingly directed in a cone in the forward direction. The evident advantage of this "backward-" or "epi-detection" is the efficient suppression of the nonresonant signal from the bulk. ${ }^{[24,27]}$ Less convenient for practical applications is the fact that the epi-directed signal strength does not depend linearly on the size but rather exhibits an oscillatory behavior. This behavior and the pronounced influence of the shape of the scattering structures on the epi-signal make the quantitative analysis of the CARS microscopic data collected in the epi-direction difficultif not impossible-in most cases.

The third possible detection geometry makes use of counterpropagating pump and Stokes beams (Figure 2). This scheme was explored both theoretically and experimentally, ${ }_{1}^{[25]}$ but was found not to offer any specific advantage over epi-detection when the image was recorded in a point-scanning manner. Counterpropagating excitation beams were, however, successfully employed in a wide-field CARS microscopy scheme. ${ }^{[28,29]}$ For this purpose, the authors illuminated a large area on top of the sample with a pump pulse employing a dark-field condenser and sent the Stokes pulse from below through the same microscope. The CARS signal was then projected onto a CCD camera. While the lateral resolution of this setup is somewhat reduced and the axial resolution is largely reduced, this experiment offers the interesting possibility to record CARS images with single laser shots and correspondingly short illumination times. This is especially attractive if excitation lasers with high pulse energies and low repetition rates are used. 
Both forward and epi-detection of CARS signals are readily implemented in commercially available laser scanning confocal microscopes. ${ }^{[30,31]}$ These offer high-frame-rate image acquisition, which is one of the most important practical requirements for many imaging applications, especially in dynamical studies of biological samples. Of special interest is the possibility to simultaneously record CARS and two-photon fluorescence images, since this combines the advantages of label-free imaging with those of the highly selective fluorescence markers based on AFPs.

Equally important in biological applications is the ability to record images with a high spatial resolution deep inside a sample. This has prompted efforts to develop CARS microscopy in two directions. The straightforward approach is to use standard microscopes possibly equipped with long-workingdistance objectives. Since forward detection of the CARS signal is often prohibited by the sample, the signals have to be collected in an epi-configuration. It has been described above that an important CARS signal in epi-direction can only be expected for structural features smaller than the excitation wavelength or from interfaces in the sample. However, the first deep-tissue-imaging CARS experiments with signal collection in the epi-direction show a strong contrast also for larger struc-

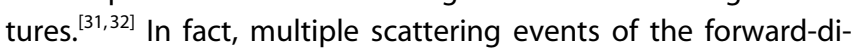
rected signal in the turbid medium allow the detection of a significant portion $(\approx 15 \%)$ of the forward signal in a backscattering geometry. ${ }^{[26]}$ It is found that because of the large size of the effective backscattering volume, the backscattering efficiency is relatively insensitive to variations in sample position. Therefore, high-quality CARS images from tissue depths of up to $\approx 200 \mu \mathrm{m}$ can be obtained in an epi-configuration. If images from deeper regions are needed, the images can be collected with an endoscope-type device. First steps towards a CARS endoscope have been published. ${ }^{[33]}$ In their prototype, the authors deliver the excitation pulses with an optical fiber. The exciting light is focused with an aspheric lens which is also used to collect the CARS signal in epi-direction. With this arrangement, it was possible to resolve submicron-sized objects. In contrast to the CARS microscopes described above, the collected light was in this case mainly the epi-signal from small structures or interfaces.

\subsection{Excitation Schemes and Sources}

Contrast generation in CARS microscopy depends on the spectroscopic selection of a proper vibrational resonance. In singlefrequency CARS microscopy, a single point in the vibrational spectrum is addressed at a time by tuning $\omega_{\mathrm{p}}-\omega_{\mathrm{s}}$ to match $\Omega_{\text {vib }}$. Information about the distribution of molecular species with different vibrational resonances can only be obtained by recording another image at a different frequency tuning. It is obvious that this scheme limits the achievable selectivity in complex samples with many different molecular components. Higher selectivity can be obtained by recording spectra over larger frequency ranges using spectral multiplexing. ${ }^{[34,35]}$ In this scheme, a long, narrow-bandwidth laser pulse serving as a pump/probe pulse is synchronized with a short, broad-band- width Stokes pulse. The interaction of both pulses leads to the probing of a wide range of vibrational levels. Dispersion of the resulting anti-Stokes signal onto a CCD camera allows the simultaneous detection of the spectral range given by the spectral convolution of the pulse widths (Figure 3). It has been

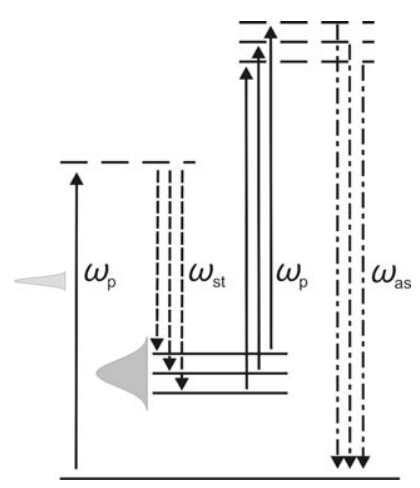

Figure 3. Multiplex CARS microscopy. A spectrally narrow pump pulse together with a spectrally broad Stokes pulse address vibrational resonances within the convoluted spectral width of the exciting lasers. Information about these resonances is obtained by dispersing the spectrally broad CARS signal.

shown that the resulting spectra can be used to identify chemical species even in congested spectral regions. ${ }^{[36,37]}$

No matter whether the images are recorded using a single frequency or a multiplexing approach, a point of major importance in CARS microscopy is the adjustment of the temporal overlap between the excitation lasers. Since many applications of CARS spectroscopy concern high-resolution studies, the lasers employed are often operated either in a continuouswave or in a pulsed mode with comparatively long pulse durations. In contrast, the broad spectral bands encountered in CARS microscopy make it necessary to use lasers that deliver pulses with durations in the fs-to-ps range in order to generate strong signals. Thus, CARS microscopy faces the same challenges as macroscopic ultrafast CARS spectroscopy or other pump/probe experiments in which two or more laser pulses have to meet at the same spot at well-defined times. ${ }^{[38]}$ In CARS microscopy, this spot is the diffraction-limited focal spot of the excitation beams.

The easiest solution to the timing problem of the two excitation pulses is the derivation of pump/probe and Stokes pulses from the same initial laser pulse. In this case, no timing jitter between the pulses will occur. Experiments of this type have been realized with several different schemes. The first CARS microscopy experiments with collinear focusing were performed with an optical parametric amplifier (OPA) laser system. A regeneratively amplified Ti:Sapphire laser provided pump and probe pulses and was used to pump an OPA, which in turn delivered the Stokes pulse. With this system, the addressable spectral range was limited to $\approx 2600-3300 \mathrm{~cm}^{-1}$. The short pulse duration of $\approx 100 \mathrm{fs}$ leads to spectrally broad pulses so that a reasonable spectral resolution can only be achieved by filtering the pulses (thereby loosing much of the pulse energy). In other early experiments, ${ }^{[22]}$ synchronously 
pumped picosecond dye lasers were used, and these suffered from significant peak-to-peak power fluctuations. Therefore, some follow-up experiments used laser equipment specifically designed to circumvent these problems.

Larger tuning ranges, better spectral resolution, and higher power stability are achieved by replacing the amplifier and OPA system by a much simpler setup in which an optical parametric oscillator (OPO) is synchronously pumped by a highpower ps-oscillator. ${ }^{[31]}$ Here, the ps-oscillator provides the Stokes beam while the frequency-doubled output of the OPO generates the pump/probe beam. Compared to the low repetition rate of amplified laser systems, the $76 \mathrm{MHz}$ repetition rate of this laser setup is also advantageous when recording fast image sequences required in many applications. OPO-based experiments have been simplified and extended in two respects: On the one hand, the signal and idler output produced in an OPO can directly be used as pump/probe and Stokes beams. For this purpose, the pump frequency of the OPO has to be shifted to the visible spectral range, since otherwise the excitation-beam frequencies lie too far in the IR region. ${ }^{[39]}$ For this type of experiment, the range of accessible low-frequency Raman transitions is only limited by the characteristics of the optical filter used to separate the excitation light from the anti-Stokes signal. This is also true for a second type of experiment, in which the signal output of two OPOs pumped in parallel is used as pump/probe and Stokes beams. While this scheme requires an additional OPO, it offers the additional possibility of performing simultaneous measurements at two frequencies. This can either be used to probe two Raman transitions or to reduce the nonresonant background. ${ }^{[40]}$

In all cases in which a single Ti:Sapphire laser is used both as pump source and for the creation of the Stokes beam in a nonlinear process, femtosecond pulses are generally being used (on the basis of efficiency arguments). Because this limits the spectral resolution in the single-frequency CARS method, various approaches using a spectral chirp on the laser pulses have been realized to achieve spectral focusing. ${ }^{[41-44]}$ In the case of multiplex CARS, the challenge is to generate a spectrally broad Stokes field from a single laser source. This has been achieved using femtosecond excitation in photonic-crystal ${ }^{[45-47]}$ and tapered fibers. ${ }^{[48]}$ Since the pump in this case is a femtosecond laser pulse either the spectral resolution must be compromised or the pump must be spectrally filtered at the expense of laser power. Alternatively, it has been demonstrated that a picosecond-amplified laser source can be used to generate a broadband Stokes field for high-spectral-resolution multiplex CARS microscopy. ${ }^{[49,50]}$

Recent advances in the electronic synchronization of ultrashort laser pulses have made it possible to solve the timing problem by externally synchronizing two independent laser oscillators. ${ }^{[51,52]}$ Although dual Ti:Sapphire laser systems with an external electronic synchronization are commercially available and are well-suited for single-frequency and multiplex CARS microscopy, these systems are still relatively complex and expensive. Therefore, several new approaches have been developed. The answer to the question regarding the optimal laser system depends on the exact application, and there is no gen- erally valid response. However, some general statements can be made: First of all, there is the issue of the wavelength range of the lasers. The ideal excitation wavelength results from a compromise between optical resolution, sampledamage threshold, and required penetration depth. The resolution increases towards shorter wavelengths. At the same time, linear absorption of the laser light by the sample should be avoided to minimize sample damage and maximize penetration depth and/or transmission. For biological samples there is a window of "minimum absorption" by water and biomolecules in the near infrared $(700-1500 \mathrm{~nm}) .{ }^{[53]}$ Second, one should consider the repetition rate of the lasers or, in other words, whether to use amplified laser systems or not. Generally speaking, amplified systems are not required and usually provide peak powers that exceed those tolerable by the specimen or induce a plethora of other nonlinear optical effects. On the other hand, a modest reduction of the repetition rate-relative to the standard $80 \mathrm{MHz}$ obtained directly from the oscillators-may prove useful to reduce optical damage. ${ }^{[54,55]}$ A pulse picker can be used to reduce the repetition rate, but more elegant methods based on passive amplification have been developed. ${ }^{[56]}$

\section{The Nonresonant Background-to Use or to Reduce?}

To generate contrast in microscopy the signal from the molecules of interest in the specimen needs to be discriminated from other contributions to the signal. The main source of background in CARS microscopy arises from the nonresonant signal. Equation (3) shows that the total CARS signal is a coherent addition of a resonant and a nonresonant contribution. The magnitude of the resonant contribution, which yields the molecular-specific contrast, is directly related to the oscillator strength and the number density of the vibrational mode (or modes) involved. As long as the spectral bandwidth of the laser pulses matches or exceeds the vibrational line width, this resonant contribution is almost independent of the laser-pulse duration. The nonresonant contribution, on the other hand, is virtually independent of the sample and increases strongly with decreasing pulse duration. As a result, the ratio of the resonant-to-nonresonant contribution decreases with decreasing pulse duration of the lasers. For transform-limited pulses this means that pulse durations of $\approx 2-3$ ps are optimal for condensed-phase applications in which vibrational bands with half-widths-at-full-maximum of $10 \mathrm{~cm}^{-1}$ are typically encountered. ${ }^{[27]}$ It was pointed out before that the coherent addition of the resonant contributions of the various vibrational modes and the nonresonant background also affects the spectral lineshape, where the vibrational resonances show apparent frequency shifts and differences in the relative peak amplitude compared to those of the spontaneous Raman spectrum. The nonresonant background is therefore an important factor in every CARS application. However, its influence depends on the mode of operation of the experiment, namely, single-frequency or multiplex CARS microscopy. 


\subsection{Single-Frequency CARS: Reduce the Nonresonant Contribution!}

In single-frequency CARS microscopy, a single point in the vibrational spectrum is addressed at a time by tuning $\omega_{\mathrm{p}}-\omega_{\mathrm{s}}$ to match the vibrational mode of interest. Tuning of the Stokes laser relative to the pump laser is required to obtain additional information about the vibrational spectrum of the sample. In practice, often an off-resonance image is acquired-at a Raman shift known to contain negligible resonant contributions-to determine the (nonresonant) background level for an image acquired on-resonance. The simultaneous use of two pump lasers allows the parallel recording of resonant and nonesonant images and the derivation of an image with a much reduced non-resonant background. ${ }^{[40]}$ In nearly all cases, the purely resonant contribution is detected on a strong nonresonant background signal. In this case, laser-induced fluctuations, such as pulse-to-pulse power fluctuations, timing jitter between the pump and Stokes laser pulses, or changes in alignment, limit the detectability of the small resonant contribution on top of the large and noisy nonresonant background. Therefore, various methods have been developed to reduce or eliminate the nonresonant contribution to the signal.

\subsubsection{Polarization CARS}

In CARS spectroscopy, the traditional method for reducing the nonresonant contribution to the CARS signal is based on using the different polarization dependencies of $\chi_{\mathrm{NR}}{ }^{(3)}$ and $\chi_{\mathrm{R}}{ }^{(3)}$. ${ }^{[57-60]}$ In this approach, different polarization angles are chosen for both the pump and the Stokes beams. The anti-Stokes signal is then selected by using an analyzer in the detection path. This configuration can be optimized either for maximum resonant signal or for maximum resonant-to-nonresonant signal. Since the angle between the resonant and the nonresonant polarization is generally very small, reducing the nonresonant contribution also leads to a considerable attenuation of the resonant contribution to the signal. It should also be realized that different vibrational modes may have different depolarization ratios, and hence require different settings of the polarizers. ${ }^{[61]}$ This fact implies that there is generally no unique polarization setting for which the dispersive character of the CARS spectrum that results from the cross term $2 \chi_{N R}{ }^{(3)} \operatorname{Re}\left\{\chi_{R}{ }^{(3)}\right\}$ is eliminated completely over the whole spectral range. This explains why this method has found only limited use in CARS microscopy ${ }_{1}^{[3,61]}$ while it is frequently employed in CARS spectroscopy. ${ }^{[62-65]}$

\subsubsection{Time-Resolved CARS Microscopy}

The resonant and nonresonant contributions to the CARS signal not only exhibit different polarization properties but they also show a different temporal behavior. This can be used to discriminate between the two. Whereas the nonresonant component of the signal is purely electronic and shows an instantaneous response to the exciting electric field, the temporal response of the resonant contribution is determined by both the vibrational lifetime $T_{1}$ and the phase-relaxation time $T_{2}$ of the vibrational modes involved. The latter properties determine the vibrational line width. Based on this different temporal behavior, the resonant part can be detected independently from the nonresonant part by means of time-resolved experiments (Figure 4). For this purpose, the pump and probe

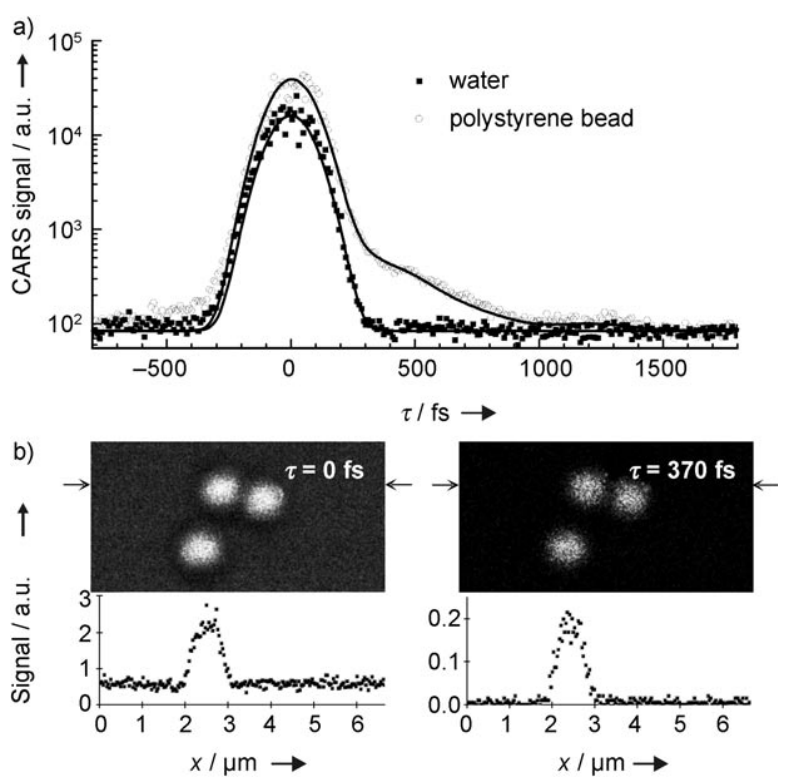

Figure 4. Suppression of the nonresonant background signal by means of time-resolved CARS microscopy of polystyrene beads with a diameter of $1 \mu \mathrm{m}$. a) Measured and simulated $(-)$ decay curves when focused on the bead $(\circ)$ and into bulk water $(\mathbf{\square})$. b) Free-induction decay images and the lateral intensity profiles along the white line at time zero (left) and at $\tau=370 \mathrm{fs}$ (right), demonstrating the complete removal of nonresonant background contributions from the sample and solvent to the image contrast in the latter. Adapted from ref. [66].

pulses are applied separately, with a tunable temporal delay between them. ${ }^{[66]}$ Similar to polarization CARS microscopy, the time-resolved detection of the CARS signal is generally accompanied by a significant attenuation of the resonant contribution. The reason for this is that since the homogeneous line widths of the vibrational resonances in the condensed phase are typically $10 \mathrm{~cm}^{-1}$ or more, most of the induced polarization decays within only a few ps. Therefore, the rejection of the first ps of the signal also suppresses most of the resonant contribution. Recently, a novel approach that uses pulse-shaping techniques to realize truly time-resolved CARS spectroscopy with a single laser was published. ${ }^{[67]}$

\subsubsection{Measurement of the Complex CARS Signal Field}

Since both polarization and time-resolved CARS microscopy have the disadvantage of severely reducing the resonant signal, alternative methods have been developed to measure separately the real and imaginary part of the CARS signal field. Whereas the nonresonant contribution contains only a real part, the resonant contribution has both a real and an imaginary part. It has been shown that the imaginary part of the reso- 
nant susceptibility $\operatorname{Im}\left\{\chi_{R}{ }^{(3)}\right\}$ is directly proportional to the spontaneous Raman-scattering spectrum. ${ }^{[36,68]}$ Separation of the real and imaginary parts can be realized by interferometric mixing of the CARS signal with a local oscillator. This local oscillator field represents a purely nonresonant CARS signal and can be obtained either separately from a nonresonant sample ${ }^{[69]}$ or from the excitation beam itself ${ }^{[70]}$ it can also be approximated by the response of the sample to broad-bandwidth-excitation pulses. ${ }^{[71]}$ Various approaches have been demonstrated both in the spectral ${ }^{[69,71-73]}$ and temporal ${ }^{[74-76]}$ domain. It has been shown that a time-gated interferometric detection of the CARS signal can be used to realize chemical specificity in optical coherence tomography (OCT) measurements. ${ }^{[77,78]}$ Inherently, axial and spectral resolution are entangled in these applications, since a large bandwidth is required for high axial resolution while the reverse is true for high spectral resolution.

\subsubsection{Other Methods}

Recently, another approach for enhancing the sensitivity to the resonant component of the signal was presented; ${ }^{[79]}$ the approach is based on a frequency modulation of the pump laser. This method uses the fact that the cross-term $2 \chi_{\mathrm{NR}}{ }^{(3)} \operatorname{Re}\left\{\chi_{\mathrm{R}}{ }^{(3)}\right\}$ has a dispersive shape. Hence, a frequency modulation around the resonant frequency translates into an amplitude modulation of the signal. The amplitude modulation can then be detected with high sensitivity using lock-in detection. This method works best for isolated vibrational resonances. Finally, epi-detection of the CARS signal should be mentioned at this point. As explained above, the CARS signal originating from features with an axial extent well below the optical wavelength, is emitted both in the forward and backward (epi) direction. The CARS signal from more extended features is emitted almost exclusively in the forward direction. Therefore, epidetection of the CARS signal strongly reduces the nonresonant contribution of the mounting medium to the CARS signal from thin features. Of course, the nonresonant contribution of these features is also emitted in both forward and backward direction.

\subsubsection{Phase and Polarization Control}

Another approach for reducing the nonresonant background in CARS microscopy is based on controlling the phase of the excitation fields present in a single-broadband laser pulse. Here, coherent-control techniques are applied to enhance the resonant contribution to the CARS signal relative to the nonresonant contribution. ${ }^{[80-82]}$ By combining phase and polarization control, background-free CARS microscopy can be realized. ${ }^{[82,83]}$ The spectral resolution in this case is constrained by the number of pixels available in the phase mask over the spectral bandwidth of the excitation. The spectral resolution achieved in this manner is better than $20 \mathrm{~cm}^{-1}$. ${ }^{83]}$ Recently, the interferometric and phase-control strategies have been combined in double-quadrature interferometric CARS. ${ }^{[84,85]}$ Here, a single phase-polarization-controlled broadband pulse is used to generate spectral interferometric traces along two polarization di- rections. The normalized difference yields the background-free multiplex CARS spectrum. Again, the effective spectral resolution and detection bandwidth are determined by the pixel limitations of the phase mask ( $30 \mathrm{~cm}^{-1}$ resolution in this case).

\subsection{Multiplex CARS: Use the Nonresonant Contribution!}

Whereas the nonresonant background limits the detection sensitivity in single-frequency CARS, it can actually be used to increase the sensitivity in multiplex CARS. In multiplex CARS, the signal is generated and detected simultaneously over a significant part of the vibrational spectrum. Therefore, the signal-tonoise ratio of the acquired CARS spectrum-and thus the detection sensitivity of the resonant spectral features therein-is not influenced by, for instance, pulse-to-pulse power fluctuations or timing jitter between the pump and Stokes pulses. Indeed, the signal-to-noise ratio is determined by the Poisson noise. ${ }^{[86,87]}$ A strong nonresonant signal reduces the relative noise and increases the resonant contribution, through the cross-term $2 \chi_{N R}{ }^{(3)} \operatorname{Re}\left\{\chi_{\mathrm{R}}{ }^{(3)}\right\}$, in a heterodyne fashion. In addition to the Poisson noise, the detection sensitivity in multiplex CARS depends on how well the shape of the nonresonant contribution can be determined. Generally, the nonresonant contribution is measured separately, for instance, by focusing in the cover glass. Once the nonresonant contribution is known, the CARS signal can be expressed as [Eq. (4)]:

$S\left(\omega_{\text {as }}\right)=\frac{\left|\chi_{R}^{(3)}\left(\omega_{\text {as }}\right)+\chi_{\mathrm{NR}}^{(3)}\right|^{2}}{\left|\chi_{\mathrm{NR}, \text { ref }}^{(3)}\right|^{2}}=\left|\chi_{\mathrm{r}}^{(3)}\left(\omega_{\mathrm{as}}\right)+\chi_{\mathrm{nr}}^{(3)}\right|^{2}$

where $S\left(\omega_{\text {as }}\right)$ is the CARS signal strength, which is independent of experimental parameters such as the laser power or the alignment. The variables $\chi_{r}^{(3)}$ and $\chi_{n r}{ }^{(3)}$ are the resonant and nonresonant contributions to the signal normalized by the nonresonant contribution of the reference $\chi_{\mathrm{NR}, \mathrm{ref}}{ }^{(3)}$. This normalization procedure makes multiplex CARS a quantitative microscopic or spectroscopic tool. ${ }^{[86,87]}$ The CARS signal strength from a particular molecule depends not only on its scattering cross-section, but also on its orientation relative to the polarization of the laser fields. Using different polarization conditions (see below), this orientation can be measured. This information can be used to translate the observed spatially resolved CARS signal strength into quantitative molecular-density maps of the specimen $^{[88]}$ (Figure 5). CARS microscopy provides chemical and physical specificity, without labeling of the specimen. Although inherently present in the vibrational spectrum, changes in the chemistry or the physics of the sample generally produce only subtle changes in the line positions, the relative amplitudes between the vibrational modes or the lineshapes. To fully exploit the CARS potential, the vibrational spectrum should be measured with a high signal-to-noise ratio; however, even then is its interpretation still not without problems. Because of the coherent addition of all the resonant contributions of the vibrational modes present and the nonresonant contribution, the CARS spectrum has a complex shape, which 
a)

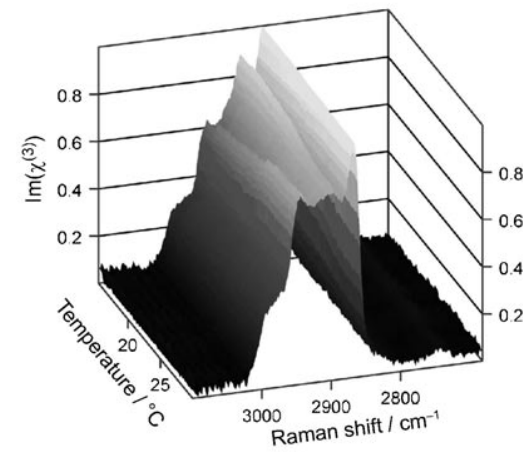

b)

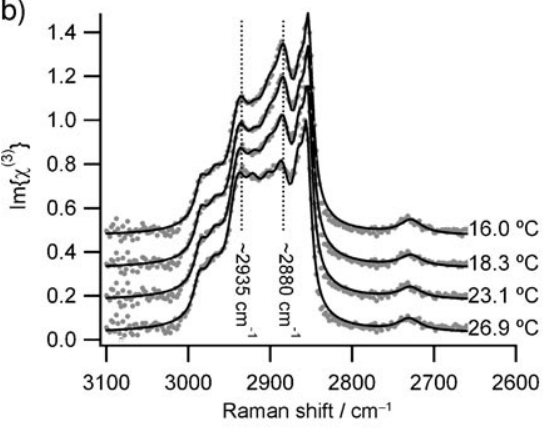

c)

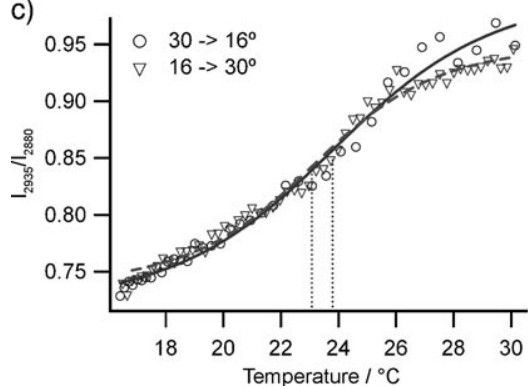

Figure 5. a) $\operatorname{Im}\left[\chi^{(3)}\right]$ spectra, retrieved from measured multiplex CARS spectra, of a $75 \mathrm{~mm}$ dimyristoylphosphatidylcholine (DMPC) small unilamellar vesicle (SUV) suspension in water as a function of temperature. The water contribution is subtracted from the $\operatorname{Im}\left[\chi^{(3)}\right]$ spectra. b) Representative $\operatorname{Im}\left[\chi^{(3)}\right]$ spectra (•) at four different temperatures and global fits (-). The curves have been off-set with increments of 0.15 for clarity. c) The ratio $I_{2935} / I_{2880}$ as a function of the temperature, as determined from the $\operatorname{Im}\left[\chi^{(3)}\right]$ spectra. The phase-transition temperatures for the $30^{\circ} \rightarrow 16^{\circ}(----)$ and $16^{\circ} \rightarrow 30^{\circ}(-)$ trace are determined from fitting with a sigmoidal-shaped function. For the maximum-entropy method, the parameter $M$ is set to its maximum value of $(K+1 / 2)(N-1)$; with $N=251, K=1$ and $M=375$. Adapted from ref. [16]

can only be readily interpreted in the case of isolated resonances. Also for multicomponent systems, this coherent addition of the different contributions precludes straightforward quantitative determination of the components present. One possibility to extract the required information from complex multiplex CARS spectra is using a least-squares fit of the theoretical expression for the CARS spectrum to the experimental data. ${ }^{[34,37,64,86,88-91]}$ This approach requires sufficient starting information about the vibrational spectrum of the sample components. Recently, it was demonstrated ${ }^{[36,37]}$ that it is also possible to extract the imaginary part of the CARS spectrum (equivalent to the spontaneous Raman spectrum) directly from the measured spectral data. This approach employs the maxi- mum entropy (ME) method to retrieve the phase function of the nonlinear susceptibility tensor elements $\chi_{i j k l}{ }^{(3)}$ responsible for the CARS lineshape. The method does not require any previous information on the vibrational-resonance structure of the sample to extract quantitative spectral data from the multiplex CARS spectra in congested spectral regions and is, in principle, applicable to any CARS spectrum of sufficient signal-to-noise ratio.

\subsection{Variants of CARS Microscopy}

\subsubsection{Single-Molecule CARS}

CARS is a coherent nonlinear optical phenomenon in which the signal amplification results from a constructive coherent addition of multiple vibrational scatterers $I_{\text {CARS }} \propto\left|\chi^{(3)}\right|^{2}$. As such it is not suitable for single-molecule-microscopy applications. However, signal enhancement also results from the third-order dependence on the laser fields $I_{\text {CARS }} \propto I_{\mathrm{p}} I_{s} I_{\text {pr }}$ Thus, increasing the laser power causes a rapid increase of the CARS signal strength. In bulk applications this approach is limited by sample damage. Alternatively, one can use the electric-field enhancement caused by surface-plasmon polariton excitations. The field enhancement is limited to the close proximity of a metallic surface (i.e. the near field) and thus circumvents sample damage. This technique is well known from surface-enhanced Raman scattering. Using $60 \mathrm{~nm}$ gold particles Hayazawa et al. ${ }^{[92]}$ succeeded in detecting a surface-enhanced Raman signal from adenine molecules attached to the gold spheres. Similarly, atomic force microscopy (AFM) can be combined with CARS microscopy, thereby using the field-enhancement effect that is observed when the excitation fields are focused on the cantilever tip. ${ }^{[93,94]}$ In another approach to realize superresolution CARS microscopy, Schaller et al. ${ }^{[95]}$ combined nearfield scanning optical microscopy (NSOM) with CARS. In this application a spatial resolution of $128 \mathrm{~nm}$ was achieved.

\subsubsection{CARS Correlation Spectroscopy}

Correlation spectroscopy is widely used to study fast dynamical processes and probe diffusional characteristics. The two major techniques employed are dynamic light scattering and fluorescence correlation spectroscopy. These correlation techniques monitor signal fluctuations that result either from changes in the number density or from spectral fluctuations; the signals are monitored within a confined probing volume. CARS can be used to add chemical selectivity to correlation spectroscopy. The probing volume is inherently confined because of the nonlinear nature of the technique. CARS correlation spectroscopy has been used to measure diffusion characteristics of polystyrene (PS) and polymethylmethacrylate (PMMA) beads of different sizes (between 110 and $528 \mathrm{~nm}^{\text {,96] }}$ see Figure 6). In similar experiments, Cheng et al. ${ }^{[89]}$ showed that an additional reduction of the nonresonant backgroundachieved by using polarization-sensitive CARS—can be applied to increase the sensitivity and selectivity of the technique in the case of Raman bands with low-mode densities. 
a)

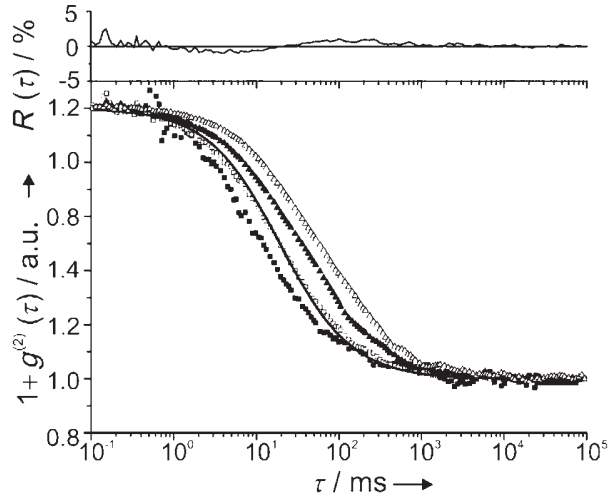

b)

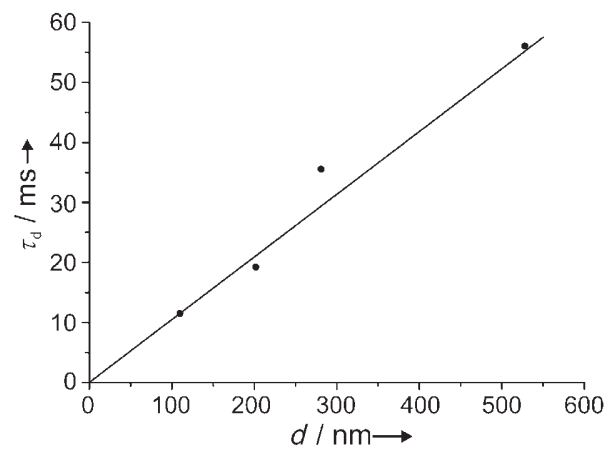

Figure 6. a) CARS correlation spectroscopy of differently sized polymer beads [polystyrene with $110(\mathbf{\bullet}), 202(\square)$, and $528 \mathrm{~nm}(\triangle)$ diameter; polymethylmetacrylate with $281 \mathrm{~nm}(\mathbf{\Delta})$ diameter] with a fit and residuals to the $202 \mathrm{~nm}$ PS data. b) Diffusion times derived from the fits to the correlation data. The line is calculated from the Stokes-Einstein equation using a diffusion time $\tau_{D}$ measured for the $110 \mathrm{~nm}$-diameter beads. Adapted from ref. [96].

\section{Applications of CARS Microscopy}

Together with the rapid development of CARS microscopy during the last decade, the first applications of this technique in biophysics and the material sciences have emerged. The majority of CARS-microscopy applications have concentrated on biological samples, with lipids as the prime topic of investigation. There are several reasons for this choice: On the one hand, they have a high scattering cross-section, are generally present in high local concentrations, and have a great significance for biology and medicine. On the other hand, the $\mathrm{C}-\mathrm{H}$ stretching vibrations of fatty acids are well separated from other resonances (this is also true for the $C-D$ stretching vibrations), which makes isotopic substitution attractive for such investigations. Lipids have been studied both in model systems and in cells.

\subsection{Lipids in Model Systems}

Lipid rafts, or lipid microdomains, are a hotly debated topic in biophysics and biology. The basic idea of this model is that lipid microdomains may occur in the plasma membrane of cells as the result of a lipid-driven phase separation. In this model, cholesterol and saturated lipids form a so-called liquidordered phase (the rafts) within a liquid-disordered sea of unsaturated lipids. Although cholesterol-induced lipid-phase separation has been demonstrated in model systems, no conclusive evidence for this phenomenon has been found in biomembranes. CARS has significant potential for unraveling the parameters that are involved in this proposed phase separation. It has been demonstrated that multiplex CARS microscopy can be used to discriminate between solid-ordered and liquid-disordered multilamellar lipid vesicles. ${ }^{[25,34]}$ Similarly, by using detailed spectral analysis of multiplex CARS on single lipid bilayers or monolayers, the lipid chain order-defined as the ratio of the signal intensity from the anti-symmetric or symmetric methylene stretching modes, respectively-could be determined quantitatively for both the liquid-disordered and the solid-ordered phases. ${ }^{[86]}$ Another strategy to distinguish between two lipid components is using deuteration. The $\mathrm{C}-\mathrm{D}$ stretching vibration is well outside the $\mathrm{C}-\mathrm{H}$ stretching region, which makes it readily detectable. Potma et al. ${ }^{[97]}$ used this strategy to visualize phase separation in giant unilamellar vesicles (GUVs) - by means of CARS—for a mixture consisting of the unsaturated lipid dioleoylphosphatidylcholine (DOPC) and a deuterated saturated lipid dipalmitoylphosphatidylcholine (DPPC). Similarly, Li et al. ${ }^{[98]}$ used deuterated DPPC to determine lipid distributions between coexisting phases in binary DOPC/d62-DPPC and ternary DOPC/d62-DPPC/cholesterol mixtures.

\subsection{Measuring the Orientational Order}

CARS can also be a powerful tool for measuring molecular orientation. This ability results from the fact that the CARS signal field $E_{\text {CARS }}$ depends on the relative orientation of the transition dipole moments $\mu_{\mathrm{ge}}$ and $\mu_{\mathrm{ev}}$ and the polarization of the laser fields, according to Equation (5):

$\mathbf{E}_{\mathrm{CARS}} \propto \sum_{i}^{N}\left(\varepsilon_{\mathrm{p}} \cdot \mu_{\mathrm{ge}}^{(i)}\right)\left(\varepsilon_{\mathrm{S}} \cdot \mu_{\mathrm{ev}}^{(i)}\right)\left(\varepsilon_{\mathrm{pr}} \cdot \mu_{\mathrm{ve}}^{(i)}\right)\left(\varepsilon_{\mathrm{as}} \cdot \mu_{\mathrm{eg}}^{(i)}\right) E_{\mathrm{p}} E_{\mathrm{s}} E_{\mathrm{pr}}$

where $i$ runs over the number of molecules (or vibrational modes) $N$ within the focal region and $E$ and $\varepsilon$ denote the amplitude and polarization of the electric fields of the pump, Stokes, and probe laser and of the anti-Stokes signal. The subscripts $g$ and $e$ denote the ground and first-excited electronicenergy levels, respectively, and $v$ denotes a ground-state vibrational level. Generally, the polarization conditions are set to fulfill the condition: $\varepsilon_{\mathrm{p}}=\varepsilon_{\mathrm{S}}=\varepsilon_{\mathrm{pr}}$ In addition, an analyzer can be used to ensure that $\varepsilon_{\mathrm{p}}=\varepsilon_{\mathrm{as}}$. Also note that $\mu_{\mathrm{ge}}=\mu_{\mathrm{eg}}$ and $\mu_{\mathrm{ev}}=$ $\mu_{\text {ve. }}$. It follows that the CARS signal strength depends on the relative orientation between the polarization of the laser field and the dipolar moments of the molecular transitions. By using this approach, the molecular orientational distribution of lipid acyl chains in multilamellar vesicles could be investigated ${ }^{[88]}$ (see Figure 7) and it was shown to depend both on the saturation level and the presence of cholesterol. With this orientational information, local lipid densities could be visualized 

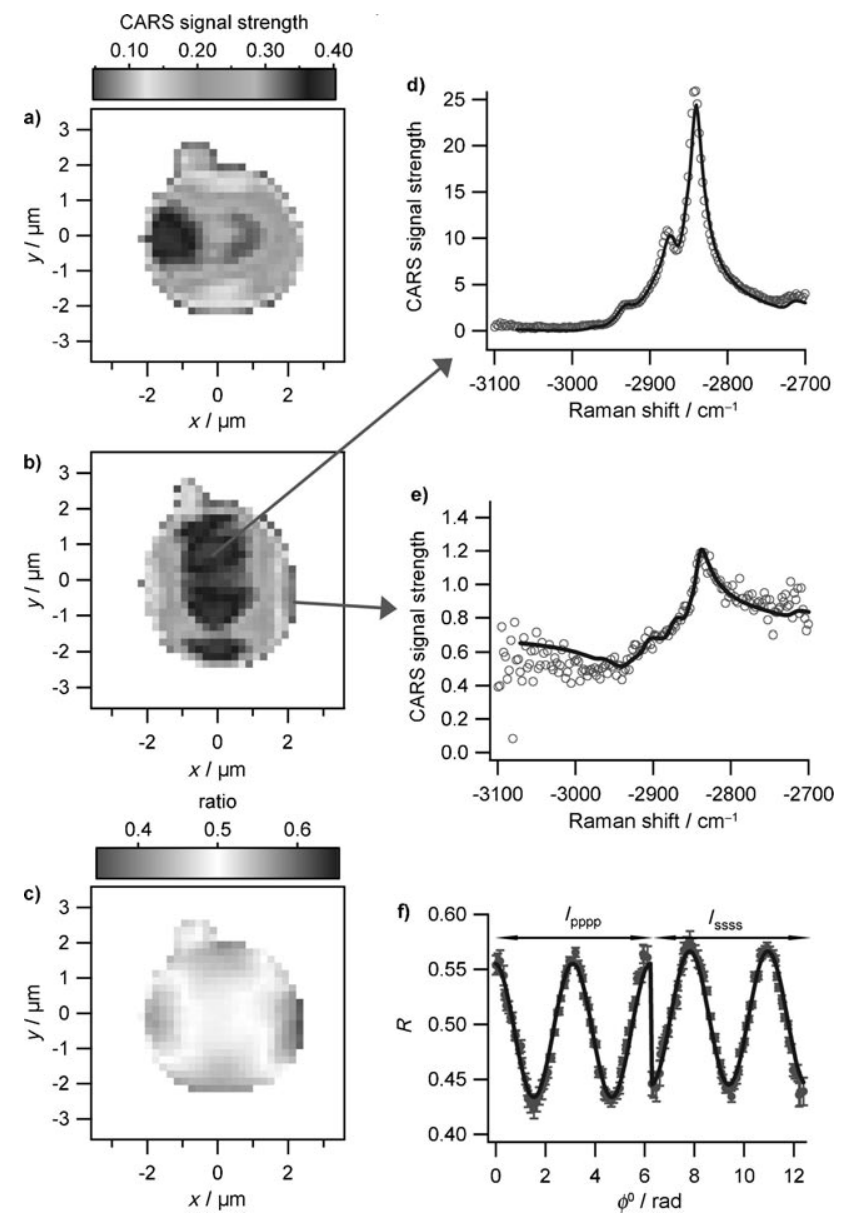

Figure 7. Multiplex CARS images of the $v_{\mathrm{s}}\left(\mathrm{CH}_{2}\right)$ mode of a DPPC multilamellar vesicle (at $2845 \mathrm{~cm}^{-1}$ ) with the polarization of all laser and signal fields in a) pppp- and (b) ssss configurations. c) Ratio image of (a) and (b)-as defined by Equation (9). d,e) Typical multiplex CARS data and fits for typical lipid spectra at maximum and minimum signal levels, respectively. f) Variation of the ratio of (c) along the rim of the vesicle and a fit to the data. Error bars denote two standard deviations. The data in (f) are fitted globally for the pppp and ssss polarization modes, as indicated by the arrows in the top of the graph. From ref. [88].

in these studies. The orientation of water molecules between closely packed lipid bilayers was investigated in a similar way. Initially, it was found ${ }^{[99]}$ that significant water ordering exists in these systems. However, Wurpel et al. ${ }^{[1]}$ showed that careful spectral evaluation is required in this case, because strong coherent interference effects between the water and lipid vibrational resonances readily lead to misinterpretation of the data. It was concluded that although a part of the (confined) interlamellar water has a weaker hydrogen-bond network than bulk water, there is negligible orientational order of the interstitial water molecules. The orientational order and the composition of surfactant lamellar phases have also been studied in myelin figures. ${ }^{[100]}$ These cylindrical tubes have long been known as a type of dynamic instability consisting of many lipid bilayers. Polarization CARS microscopy shows that while some partially ordered water is found between the bilayers, corelike water structures are found within the myelin figures.

\subsection{Biological Applications}

A major motivation for the development of CARS microscopy is its application in investigating biological problems. In these experiments, the specific advantages of CARS microscopy are fully exploited. No labeling is necessary (which allows imaging under physiological conditions), and the fact that the contrast does not degrade by means of photobleaching is used in long-term imaging studies.

The first biological application of CARS microscopy was the observation of water diffusion in live Dictyostelium cells. ${ }^{[101]}$ Water has a broad vibrational resonance centered at $3300 \mathrm{~cm}^{-1}$, which is shifted to approximately $2700 \mathrm{~cm}^{-1}$ in $\mathrm{D}_{2} \mathrm{O}$. Since the bands have a width of several hundred wavenumbers, fs laser excitation yields intense CARS signals of water. Water diffusion in live cells is observed by flushing the aqueous environment of the cells with $\mathrm{D}_{2} \mathrm{O}$. Fast-line scanning of the cells showed that a significant region close to the plasma membrane is characterized by restricted water diffusion. A detailed analysis gives access to the membrane permeabilities. Already in these early experiments it was realized that CARS microscopy could be readily combined with simultaneous two-photon-excitation fluorescence microscopy. While the simultaneous observation of fluorescent lifetime changes validated the results obtained from CARS microscopy, it is obvious that the latter elegantly circumvents possible problems that may arise when using fluorophores (e.g. the incomplete cell entry of the marker dye or its unspecific binding to cellular structures).

In the example described above, dynamic imaging was only possible by using a line-scanning approach. Since many cellular processes take place on a subsecond timescale, microscopy techniques with a corresponding temporal resolution are needed. This problem was solved by using a modified confocal microscope ${ }^{[30]}$ for CARS microscopy. With such a setup it is possible to image the chromosome distribution during mitosis using the symmetric stretching vibration of the DNA phosphate backbone.

Because of their good detectability, the observation of lipids is also preferred in CARS microscopy experiments of live cells. The sensitivity of CARS microscopy is high enough to detect even lipid vesicles with sizes smaller than $300 \mathrm{~nm}$ in diame$\operatorname{ter}^{[102]}$ (these are hardly observed by using differential interference contrast). Fluorescence staining and fixation of the cells is also possible, but this method is prone to induce the aggregation of vesicles. ${ }^{[103]}$ CARS microscopy could confirm that the surfactants used for the dissolution of the fluorescent dyes may lead to the aggregation of lipid vesicles and to important changes in their morphology. In contrast to fluorescence microscopy, CARS allows long-term investigations of cell differentiation that extend over $192 \mathrm{~h}$. The absence of photobleaching is an equally important advantage if fast intracellular dynamics are to be followed. For this purpose, high frame rates are necessary, which means that the sample is more or less permanently illuminated. In fluorescence microscopy, this would lead to very short overall observation times caused by photobleaching. The high potential of CARS microscopy for such applica- 
tions has been exemplified by tracking lipid droplets (LD) in live cells. ${ }^{[54]}$ Whereas the biological importance of LDs in cellular processes has recently been recognized, there is only little information on their intracellular trafficking. Tracking studies with CARS microscopy revealed that LDs are actively transported along microtubuli in Y-1 cells. Simultaneous CARS and twophoton microscopy studies further suggest that the important interactions between LDs and the mitochondria in cells are mainly regulated by the motility of the LDs and not by the density of the interaction partners.

A logical extension of these first applications of CARS microscopy in cell microscopy are live animals experiments. Also here, the main focus has been given to the detection of $\mathrm{C}-\mathrm{H}$ stretching vibrations of fatty acids either in mice ${ }^{[31,104]}$ or in the model organism C. elegans ${ }^{[40]}$ (see Figure 8). An obvious prob- a)

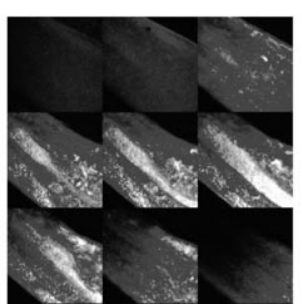

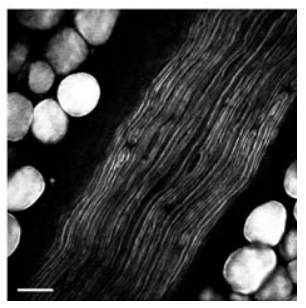

Figure 8. a) Three-dimensional CARS imaging of live C. elegans. The distance between the images (from upper left to lower right) is $2 \mu \mathrm{m}$, each image has a size of $50 \times 50 \mu \mathrm{m}^{2}$. The image acquisition time was $20 \mathrm{~s}$. Adapted from ref. [40]. b) Epi-detected CARS image of parallel myelinated axons in the sciatic nerve and the surrounding fat cells. Scale bar $=25 \mu \mathrm{m}$. Adapted from ref. [104].

lem met in experiments with larger animals or with thick tissue slices $^{[32]}$ is the excitation geometry. As has been outlined, CARS signals from larger structural sample features are preferably detected in the forward direction. This, however, is not possible if larger animals are investigated, where only an epi-detection scheme can be employed. Fortunately, it turns out that this scheme is rather efficient in collecting the CARS signals. The underlying mechanism for this unexpected observation is multiple backscattering of the forward-directed CARS signal. ${ }^{[54]}$ CARS microscopy can therefore be used to give detailed images of the lipid distribution in $\mathrm{skin}^{[31]}$ or of the myelin sheath covering spinal tissue either "ex vivo" or "in vivo". ${ }^{32,104]}$ In the latter case, CARS microscopy gives detailed insight into the organization of the myelin sheaths. A major concern in nonlinear optical experiments with live cells, functional tissue slices, or live animals is photodamage caused by the pulsed laser excitation. In general, photodamage with a linear and a nonlinear intensity dependence can be distinguished. The excitation conditions chosen for CARS microscopy are comparable to those of two-photon fluorescence excitation microscopy. The difference concerns the fact that a two-photon electronic resonance is now replaced by a Raman resonance. In general, the averaged excitation power gives rise to the linear photodamage effects whereas the pulse energies are responsible for nonlinear photodamage. From careful investigations of the dependence of photodamage on both these factors and on the excitation wavelengths, ${ }^{[31,55]}$ the following picture emerges: Average powers of approximately $10 \mathrm{~mW}$ do not induce any palpable photodamage, even under prolonged illumination. The corresponding limit for the pulse energies lies around $2 \mathrm{~nJ}$. In general, red-shifted excitation appears to be more benign, but care must be taken here since water absorptions above $840 \mathrm{~nm}$ might change this picture for certain samples. Interestingly, tuning of the excitation lasers to a Raman resonance also leads to slight increase of the photodamage. ${ }^{[105]}$

\subsection{Material Science Applications}

The study of biological samples was the main focus of the early CARS microscopy applications presented above. However, the technique has an equally high potential for investigating samples with material-scientific importance. This is especially true when the sample has a high background-fluorescence level, which impedes the use of Raman excitation. A major advantage of most of the material scientific samples is the fact that the chemical composition of the samples is usually wellknown. It is therefore easy to distinguish different components on the basis of a few selected vibrational resonances (Figure 9). CARS microscopy has been used in the imaging of lithographically imprinted patterns of polymer photoresist. ${ }^{[106]}$ In this example, the authors converted a tert-butoxyl carbonyl group of the photoresist into a hydroxy group. With the conversion process they generated a line pattern in the sample (with a spacing of $\approx 250 \mathrm{~nm}$ and consisting of converted and nonconverted areas). In the vibrational spectrum of the converted material, a skeletal mode of the tert-butoxyl group (with a resonance of $880 \mathrm{~cm}^{-1}$ ) is missing. CARS microscopy therefore allowed a clear distinction between converted and nonconverted material in the sample (Figure 9). Also in this material-scientific application, the chemical specificity, the high spatial resolution $(\approx 270 \mathrm{~nm})$, and the short acquisition times make CARS a very attractive alternative to the established techniques. These features have recently been exploited in investigations of the vibrational properties of porphyrin J-aggregate microcrystals, ${ }^{[107]}$ but also of crystalline and biological surfaces. ${ }^{[108]}$ The absence of photobleaching, as the other big advantage of CARS microscopy, can be tremendously useful in material science. This is exemplified in a recent study of repulsive and attractive interactions of colloidal particles during glass formation. ${ }^{[109]}$ While dynamic light scattering offers insight into this type of problem, more details can be obtained by directly analyzing the motion of particles captured in videos. This can be done by using fluorescence microsco$\mathrm{py}_{1}^{[110,111]}$ but here photobleaching is a problem, since long observation times are necessary. With CARS microscopy it was possible to study micron-sized unlabelled beads for more than 10000 s. $^{[109]}$

\section{Outlook}

The preceding sections have presented CARS microscopy as a new nonlinear optical technique. The combination of vibrational spectroscopy and microscopy allows highly sensitive investi- 
a)
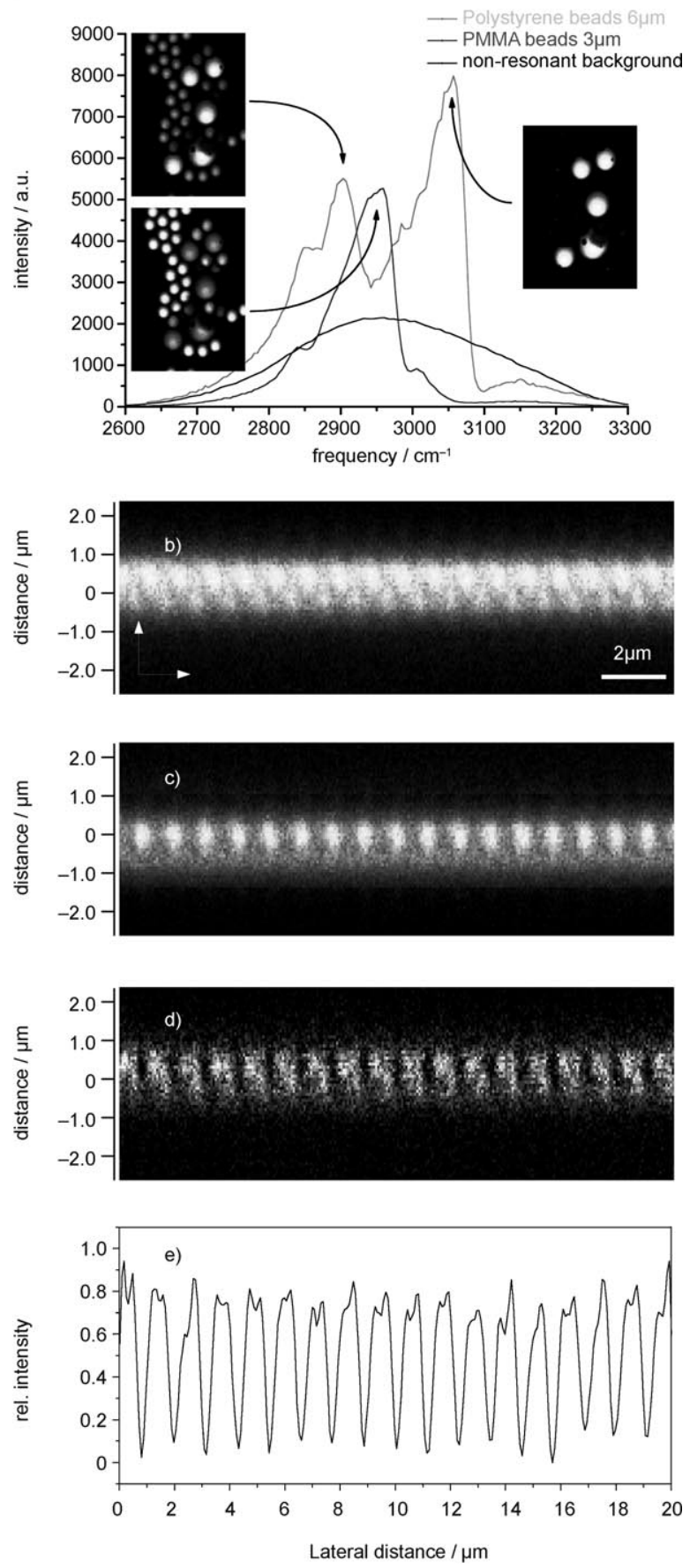

Figure 9. a) Spectrally focused CARS microscopy of PMMA (3 $\mu$ m diameter) and polystyrene ( $6 \mu \mathrm{m}$ diameter) beads. Both materials can be distinguished by their vibrational resonances. b-e) Epi-detected CARS microscopy of a patterned photoresist film. Depth-resolved $x z$ images recorded at Raman shifts of on-resonance with the nonconverted material at $880 \mathrm{~cm}^{-1}$ (b) and off-resonance at $925 \mathrm{~cm}^{-1}$ (c). The difference image and its profile plot are depicted in (d) and (e), respectively. [Figures (b)-(e) were adapted from ref. [106]].

gations of unlabelled samples in which contrast is generated with molecular specificity. These features make it an ideal tool for studies of a broad variety of samples. The main drawback of CARS microscopy is the fact that it is not a zero-background technique. Instead, the signal has to be detected against a nonresonant background. The need to solve this problem is reflected in the rapid technological development observed during the last decade, which led to many different experimental realizations of CARS-microscopy schemes. In the meantime, these experiments have reached such a level that the first applications of CARS microscopy are being published. From these results it is obvious that CARS microscopy has the potential to become an important complementary technique for other well-established microscopic methods. Although it has some limitations, it can offer a unique access to many problems which cannot be tackled with conventional techniques. For this reason, it can be expected that the impressive growth of the field will continue, with a focus on novel applications that range from microspectrosopy in advanced materials to the imaging of live biological cells.

Keywords: CARS (coherent anti-Stokes Raman scattering) fluorescence • nonlinear optics • optical spectroscopy resonance

[1] J. B. Pawley, Handbook of Biological Confocal Microscopy, 3rd ed., Springer, Berlin, 2006.

[2] R. Y. Tsien, Annu. Rev. Biochem. 1998, 67, 509-544.

[3] Ph. Tamarat, A. Maali, B. Lounis, M. Orrit, J. Phys. Chem. A 2000, 104, $1-16$.

[4] W. E. Moerner, J. Phys. Chem. B 2002, 106, 910-927.

[5] P. Tinnefeld, M. Sauer, Angew. Chem. 2005, 117, 2698-2728; Angew. Chem. Int. Ed. 2005, 44, 2642-2671.

[6] G. T. Boyd, Y. R. Shen, T. Hänsch, Opt. Lett. 1986, 11, 97-99.

[7] L. Canioni, S. Rivet, L. Sager, R. Barille, P. Vacher, P. Voisin, Opt. Lett. 2001, 26, 515-517.

[8] P. J. Campagnola, L. Löw, Nat. Biotechnol. 2003, 21, 1356-1360.

[9] Y. Barad, H. Eisenberg, M. Horowitz, Y. Silberberg, Appl. Phys. Lett. 1997, 70, $922-924$.

[10] J. A. Squier, M. Müller, G. J. Brakenhoff, Opt. Express 1998, 3, 315-324.

[11] D. Débarre, W. Supatto, A.-M. Pena, A. Fabre, T. Tordjmann, L. Combettes, M.-C. Schanne-Klein, E. Beaurepaire, Nat. Methods 2006, 3, 4753.

[12] Raman Microscopy: Developments and Applications (Eds.: G. Turrell, J. Corset), Elsevier, San Diego, 1996.

[13] J.-X. Cheng, X. S. Xie, J. Phys. Chem. B 2004, 108, 827-840.

[14] A. Volkmer, J. Phys. D 2005, 38, R59-R81.

[15] Y. R. Shen, The Principles of Nonlinear Optics, 1st ed., Wiley, New York, 1984.

[16] H. A. Rinia, M. Bonn, M. Müller, E. M. Vartiainen, ChemPhysChem 2007, 8, 279-287.

[17] S. Mukamel, Principles of Nonlinear Optical Spectroscopy, 1st ed., Oxford University Press, Oxford, 1995.

[18] W. Denk, J. H. Strickler, W. W. Webb, Science 1990, 248, 73-76.

[19] H. Lotem, R. T. Lynch, Jr., N. Bloembergen, Phys. Rev. A 1976, 14, 1748 1755.

[20] P. D. Maker, R. Terhune, Phys. Rev. 1965, 137, $801-818$.

[21] M. D. Duncan, J. Reintjes, T. J. Manuccia, Opt. Lett. 1982, 7, 350-352.

[22] M. Müller, J. A. Squier C. A. de Lange, G. J. Brakenhoff, J. Microsc. 2000, $197,150-158$.

[23] A. Zumbusch, G. R. Holtom, X. S. Xie, Phys. Rev. Lett. 1999, 82, 4142 4145.

[24] A. Volkmer, J.-X. Cheng, X. S. Xie, Phys. Rev. Lett. 2001, 87, 023901.

[25] J.-X. Cheng, A. Volkmer, X. S. Xie, J. Opt. Soc. Am. B 2002, 19, $1363-$ 1375.

[26] N. Djaker, D. Gachet, N. Sandeau, P.-F. Lenne, H. Rigneault, Appl. Opt. 2006, 45, 7005-7011.

[27] J.-X. Cheng, A. Volkmer, L. D. Book, X. S. Xie, J. Phys. Chem. B 2001, 105, 1277-1280. 
[28] C. Heinrich, S. Bernet, M. Ritsch-Marte, Appl. Phys. Lett. 2004, 84, 816818.

[29] C. Heinrich, S. Bernet, M. Ritsch-Marte, New J. Phys. 2006, 8, 36

[30] J.-X. Cheng, Y. K. Jia, G. Zheng, X. S. Xie, Biophys. J. 2002, 83, 502-509.

[31] C. L. Evans, E. O. Potma, M. Puorishaag, D. Côté, C. P. Lin, X. S. Xie. , Proc. Natl. Acad. Sci. USA 2005, 102, 16807-16812.

[32] H. Wang, Y. Fu, P. Zickmund, R. Shi, J.-X. Cheng, Biophys. J. 2005, 89, $581-591$.

[33] F. Légaré, C. L. Evans, F. Ganikhanov, X. S. Xie, Opt. Express. 2006, 14 4427-4432

[34] M. Müller, J. M. Schins, J. Phys. Chem. B 2002, 106, 3715-3723.

[35] J.-X. Cheng, A. Volkmer, L. D. Book, X. S. Xie, J. Phys. Chem. B 2002, $106,8493-8498$.

[36] E. M. Vartiainen, H. A. Rinia, M. Müller, M. Bonn, Opt. Express 2006, 14 $3622-3630$.

[37] H. A. Rinia, M. Bonn, M. Müller, J. Phys. Chem. B 2006, 110, $4472-4479$.

[38] R. Leonhardt, W. Holzapfel, W. Zinth, W. Kaiser, Chem. Phys. Lett. 1987, $133,373-377$

[39] F. Ganikhanov, S. Carrasco, X. S. Xie, M. Katz, W. Seitz, D. Kopf, Opt Lett. 2006, 31, 1292-1294,

[40] O. Burkacky, A. Zumbusch, C. Brackmann, A. Enejder, Opt. Lett. 2006 31, 3656-3658.

[41] Th. Hellerer, A. M. K. Enejder, A. Zumbusch, Appl. Phys. Lett. 2004, 85, $25-27$.

[42] K. P. Knutsen, J. C. Johnson, A. E. Miller, P. B. Petersen, R. J. Saykally, Chem. Phys. Lett. 2004, 387, 436-441.

[43] R. Porter, F. Shan, T. Guo, Rev. Sci. Instrum 2005, 76, 043108.

[44] K. P. Knutsen, B. M. Messer, R. M. Onorato, R. J. Saykally, J. Phys. Chem B 2006, 110, 5854-5864.

[45] H. N. Paulsen, K. M. Hilligsøe, J. Thøgersen, S. R. Keiding, J. J. Larsen, Opt. Lett. 2003, 28, 1123-1125.

[46] E. R. Andresen, S. R. Keiding, E. O. Potma, Opt. Express 2006, 14, 7246 7251.

[47] H. Kano, H. Hamaguchi, Appl. Phys. B 2005, 80, 243-246.

[48] T. W. Kee, M. T. Cicerone, Opt. Lett. 2004, 29, 2701-2703.

[49] V. V. Yakovlev, J. Raman Spectrosc. 2003, 34, 957-964.

[50] G. I. Petrov, V. V. Yakovlev, Opt. Express 2005, 13, 1299-1306.

[51] E. O. Potma, D. J. Jones, J.-X. Cheng, X. S. Xie, J. Ye, Opt. Lett. 2002, 27, $1168-1170$.

[52] D. J. Jones, E. O. Potma, J.-X. Cheng, B. Burfeindt, Y. Pang, X. S. Xie, Rev. Sci. Instrum. 2002, 73, 2843-2848.

[53] K. Svoboda, S. M. Block, Annu. Rev. Biophys. Biomol. Struct. 1994, 23, $247-285$.

[54] X. Nan, E. O. Potma, X. S. Xie, Biophys. J. 2006, 91, 728-735.

[55] Y. Fu, H. Wang, R. Shi, J.-X. Cheng, Opt. Express 2006, 14, 3942-3951.

[56] E. O. Potma, X. S. Xie, J. Raman Spectrosc. 2003, 34, 642-650.

[57] M. A. Yuratich, D. C. Hanna, . Mol. Phys. 1977, 33, 671-682.

[58] S. A. Akhmanov, A. F. Bunkin, S. G. Ivanov, N. J. Koroteev, Sov. Phys. JETP 1978, 47, 667-677.

[59] M. D. Levenson, G. L. Eesley, Appl. Phys. 1979, 19, 1-17.

[60] M. D. Levenson, J. Raman Spectrosc. 1981, 10, 9-23.

[61] J.-X. Cheng, L. D. Book, X. S. Xie, Opt. Lett. 2001, 105, 1341 - 1343.

[62] A. Voroshilov, G. W. Lucassen, C. Otto, J. Greve, J. Raman Spectrosc. $1995,26,443-450$

[63] A. Voroshilov, Polarised Coherent Raman and Saturation Micro-Raman Studies of Biological Macromolecules, Technical University Twente (NL), 1997.

[64] C. Otto, A. Voroshilov, S. G. Kruglik, J. Greve, J. Raman Spectrosc. 2001, 32, 495-501.

[65] J. Popp, J. Koster, S. Schlücker, J. Raman Spectrosc. 2006, 37, 384-391.

[66] A. Volkmer, L. D. Book, X. S. Xie, Appl. Phys. Lett. 2002, 80, 1505-1507.

[67] B. von Vacano, M. Motzkus, Opt. Commun. 2006, 264, 488-493.

[68] R. W. Hellwarth, Prog. Quantum Electron. 1977, 5, 1-68.

[69] C. L. Evans, E. O. Potma, X. S. Xie, Opt. Lett. 2004, 29, 2923-2925.

[70] B. von Vacano, T. Buckup, M. Motzkus, Opt. Lett. 2006, 31, 2495-2497.

[71] T. W. Kee, H. Zhao, M. T. Cicerone, Opt. Express 2006, 14, 3631-3640.
[72] E. O. Potma, C. L. Evans, X. S. Xie, Opt. Lett. 2006, 31, 241-243.

[73] M. Cui, M. Joffre, J. Skodack, J. P. Ogilvie, Opt. Express 2006, 14, 8448 8458 ,

[74] D. L. Marks, S. A. Boppart, Phys. Rev. Lett. 2004, 92, 123905.

[75] J. P. Ogilvie, E. Beaurepaire, A. Alexandrou, M. Joffre, Opt. Lett. 2006, $31,480-482$.

[76] M. Greve, B. Bodermann, H. R. Telle, P. Baum, E. Riedle, Appl. Phys. B $2005,81,875-879$.

[77] C. Vinegoni, J. S. Bredfeldt, D. L. Marks, S. A. Boppart, Opt. Express 2004, 12, $331-341$.

[78] J. S. Bredfeldt, C. Vinegoni, D. L. Marks, S. A. Boppart, Opt. Lett. 2005, 30, 495-497.

[79] F. Ganikhanov, C. L. Evans, B. G. Saar, X. S. Xie, Opt. Lett. 2006, 31, $1872-1874$

[80] N. Dudovich, D. Oron, Y. Silberberg, Nature 2002, 418, 512-514.

[81] D. Oron, N. Dudovich, D. Yelin, Y. Silberberg, Phys. Rev. Lett. 2002, 88, 063004.

[82] D. Oron, N. Dudovich, D. Yelin, Y. Silberberg, Phys. Rev. A 2002, 65, 043408.

[83] D. Oron, N. Dudovich, Y. Silberberg, Phys. Rev. Lett. 2003, 90, 213902.

[84] S.-H. Lim, A. G. Caster, S. R. Leone, Phys. Rev. A 2005, 72, 041803.

[85] S.-H. Lim, A. G. Caster, O. Nicolet, S. R. Leone, J. Phys. Chem. B 2006, $110,5196-5204$

[86] G. W. H. Wurpel, J. M. Schins, M. Müller, J. Phys. Chem. B 2004, 108, $3400-3403$.

[87] M. Müller, J. M. Schins, G. W. H. Wurpel, J. Phys. Chem. B 2004, 106, $3715-3723$.

[88] G. W. H. Wurpel, H. A. Rinia, M. Müller, J. Microsc. 2005, 218, 37-45.

[89] J.-X. Cheng, E. O. Potma, X. S. Xie, J. Chem. Phys. A 2002, 106, 8561 8568.

[90] G. W. H. Wurpel, J. M. Schins, M. Müller, Opt. Lett. 2002, 27, 1093-1095.

[91] G. W. H. Wurpel, M. Müller, Chem. Phys. Lett. 2006, 425, 336-341.

[92] N. Hayazawa, T. Ichimura, M. Hashimoto, Y. Inouye, S. Kawata, J. Appl. Phys. 2004, 95, 2676-2681.

[93] T. Ichimura, N. Hayazawa, M. Hashimoto, Y. Inouye, S. Kawata, Phys. Rev. Lett. 2004, 92, 220801.

[94] T. Ichimuraand N. Hayazawa, M. Hashimoto, Y. Inouye, S. Kawata, Appl. Phys. Lett. 2006, 84, 1768-1770.

[95] R. D. Schaller, J. Ziegelbauer, L. F. Lee, L. H. Haber, R. J. Saykally, J. Phys. Chem. B 2002, 106, 8489-8492.

[96] Th. Hellerer, A. Schiller, G. Jung, A. Zumbusch, ChemPhysChem 2002, 3, 630-634.

[97] E. O. Potma, X. S. Xie, ChemPhysChem 2005, 6, 77-79.

[98] L. Li, H. Wang, J.-X. Cheng, Biophys. J. 2005, 89, 3480-3490.

[99] J.-X. Cheng, S. Pautot, D. A. Weitz, X. S. Xie, Proc. Natl. Acad. Sci. USA 2003, 100, 9826-9830.

[100] A. P. Kennedy, J. Sutcliffe, J.-X. Cheng, Langmuir 2005, 21, 6478-6486.

[101] E. O. Potma, W. P. de Boeij, P. J. M. van Haastert, D. A. Wiersma, Proc. Natl. Acad. Sci. USA 2001, 98, 1577-1582.

[102] X. Nan, J.-X. Cheng, X. S. Xie, Biophys. J. 2003, 91, 728-735.

[103] S. Fukumoto, T. Fujimoto, Histochem. Cell Biol. 2002, 118, 423-428.

[104] T. B. Huff, J. X. Cheng, J. Microsc. 2007, 225, 175-182.

[105] H. Wang, Y. Fu, J.-X. Cheng, J. Opt. Soc. Am. B 2007, 24, 544-552.

[106] E. O. Potma, X. S. Xie, L. Muntean, J. Preusser, D. Jones, J. Ye, S. R. Leone, W. D. Hinsberg, W. Schade, J. Phys. Chem. B 2004, 108, $1296-$ 1301.

[107] H. Kano, H. Hamaguchi, Vib. Spectrosc. 2006, 42, 135-139.

[108] H. Kano, H. Hamaguchi, Chem. Lett. 2006, 1039-1047.

[109] L. J. Kaufman, D. A. Weitz, J. Chem. Phys. 2006, 125, 074716.

[110] W. K. Kegel, A. van Blaaderen, Science 2000, 287, 290-293.

[111] E. R. Weeks, J. C. Crocker, A. C. Levitt, A. Schofield, D. A. Weitz, Science $2000,287,627-631$ 